\title{
Synthesis of Novel Benzylic 1,2,3-triazole-4-carboxamides and their in vitro Activity Against Clinically Common Fungal Species
}

\author{
Ricardo García-Monroy, ${ }^{1,2}$ Davir González-Calderón, ${ }^{1 *}$ Alejandra Ramírez-Villalva, ${ }^{1,2}$ Salvador \\ Mastachi-Loza, ${ }^{1,2}$ José G. Aguirre-de Paz, ${ }^{1}$ Aydee Fuentes-Benítes ${ }^{1}$, Carlos González-Romero ${ }^{1 *}$ \\ ${ }^{1}$ Departamento de Química Orgánica, Facultad de Química, Universidad Autónoma del Estado de México, \\ Paseo Colón/Paseo Tollocan s/n, Toluca, Estado de México, 50120, México. \\ ${ }^{2}$ Departamento de Microbiología, Facultad de Química, Universidad Autónoma del Estado de México, Paseo \\ Colón/Paseo Tollocan s/n, Toluca, Estado de México, 50120, México.
}

*Corresponding author: Davir González-Calderón, email: qfb_dgonzalez@yahoo.com.mx; tel.: +52 722 2175109 ext113; fax: +52 7222173890 .

Received October $6^{\text {th }}, 2020$; Accepted January $11^{\text {th }}, 2021$.

DOI: http://dx.doi.org/10.29356/jmcs.v65i2.1457

\begin{abstract}
A library of novel benzylic 1,2,3-triazole-4-carboxamides (3a-m) were obtained with acceptable yields via a one-pot procedure. The series of compounds was screened for fungicidal activity and evaluated in vitro against four filamentous fungi and four Candida species. The former consisted of Aspergillus fumigatus, Trichosporon cutaneum, Rhizopus oryzae and Mucor hiemalis, and the latter C. krusei, C. albicans, C. utilis and $C$. glabrata. According to the in vitro assays, $\mathbf{3 d}$ and $\mathbf{3 e}$ were the most efficient fungicidal agents (of all the test compounds) against $R$. oryzae, even better than the reference drug (itraconazole). Thus, 3d and 3e represent important scaffolds that can be modified to increase antifungal activity. Additionally, they are candidates for complementary studies on the inhibition of clinical infections produced by Rhizopus spp. strains.
\end{abstract}

Keywords: 1,2,3-Triazoles, antifungal activity, 1,3-dipolar cycloaddition, Rhizopus oryzae.

Resumen. Se obtuvo una librería de nuevos bencil 1,2,3-triazoles-4-carboxamidas (3a-m) con rendimientos aceptables mediante un procedimiento one-pot. La serie de compuestos se seleccionó para determinar la actividad fungicida llevando a cabo una evaluación in vitro contra cuatro hongos filamentosos y cuatro especies de Candida. Los primeros consistieron en Aspergillus fumigatus, Trichosporon cutaneum, Rhizopus oryzae y Mucor hiemalis, mientras que para las segundas especies, esta fueron $C$. krusei, C. albicans, C. utilis y $C$. glabrata. Según los ensayos in vitro, 3d y 3e fueron los agentes fungicidas más eficaces (de todos los compuestos de prueba) contra $R$. oryzae, incluso mejores que el fármaco de referencia (itraconazol). Por tanto, 3d y 3e representan importantes núcleos que podrían modificarse para aumentar la actividad antifúngica, siendo excelentes candidatos para estudios complementarios sobre la inhibición de infecciones clínicas producidas por Rhizopus spp.

Palabras clave: 1,2,3-triazol, actividad antifúngica, cicloadición 1,3-dipolar, Rhizopus oryzae. 


\section{Introduction}

Viral, bacterial, and parasitic diseases have profoundly harmed the health of millions of people at different times in history. Such diseases include Black Death (yersinia pestis), cholera (vibrio cholerae), malaria (plasmodium), and smallpox. More recently, HIV, dengue and coronavirus have appeared on the scene. However, fungal infections have rarely resulted in serious diseases, at least during the recorded history of human beings. However, there is something uniquely problematic about fungi. Unlike bacteria, they are eukaryotes and thus share many similarities with the cells of their human hosts. Whereas antibiotics only target prokaryotic cells, compounds that kill fungi also harm the eukaryotic host, which impairs the development of antifungal agents and makes these infections the most difficult to treat. Furthermore, fungal tropism is highly variable, as pathogens infect a wide range of cell types. Depending on the immunological status of the host, a single fungal pathogen may infect multiple tissues in the same patient [1].

Among the estimated 1.5-5 million fungal species on the planet, those able to cause disease in humans are only a few hundred. Of these, a small number fulfill the four basic conditions necessary to affect healthy people: high temperature tolerance, ability to invade the human host, lysis and absorption of human tissue, and resistance to the human immune system. It is unusual for fungal disease to take hold in healthy individuals because the immune system of humans (and animals) is sophisticated, having evolved in constant response to fungal challenges. In contrast, fungal diseases occur frequently in immunocompromised patients. The four major fungal phyla that infect humans are Entomophthoromycota (Conidiobolus spp. and Basidiobolus spp.), Ascomycota (e.g. Candida spp. Fusarium spp., Histoplasma spp., Aspergillus spp., Coccidioides spp., and Pneumocystis spp.), Basidiomycota (Cryptococcus spp. and Trichosporon spp.) and Mucorales (Mucor spp. and Rhizopus spp.). [2-3]

Due to the increasing rate of fungal infections in hospitalized and immunocompromised patients, there is an urgent need to discover new antimycotic drugs. The biological attributes of triazole scaffolds, including those of the 1,2,3- and 1,2,4-isomers, are well recognized in the field of medicinal chemistry [4-10]. Many FDA-approved drugs contain such cores, being more common those with the 1,2,3-isomer: tazobactam and cefatrizine (broad-spectrum antibacterial agents), rufinamide (an anticonvulsant), suvorexant (a medication for insomnia), ticagrelor (a treatment to prevent stroke, heart attack and other adverse events in people with acute coronary syndrome) and bisoctrizole (a broad-spectrum chromophore added to sunscreens) (Scheme 1). Regarding conventional chemotherapy treatments for invasive, mucosal, and superficial fungal infections, the field is dominated by the 1,2,4-isomer. Itraconazole, terconazole, fluconazole and posaconazole (among others) have been conventional 1,2,4-triazole fungicidal agents for over 30 years, while efinaconazole and isavuconazole were more recently approved by the FDA (in 2014 and 2015, respectively) [11-17].

For pathogenic fungi, as with all microorganisms, there is the specter of the emergence of strains resistance to pharmaceuticals [18-21], particularly those observed for Rhizopus (mucormycosis) as 1,2,4triazole-drug resistant pathogen [22-23]. To meet this challenge, it is necessary to design and develop drugs that have well-defined and fungal-specific targets. The principal molecular target of azole antifungals is a protein, known as Erg11p or Cyp51p (according to distinct gene-based nomenclatures), in cytochrome P450. Cyp51p catalyzes the oxidative removal of the 14a-methyl group of lanosterol and/or eburicol in fungi by monooxygenase activity typical of P450. It contains an iron protoporphyrin moiety located at the active site. A bond is formed between a nitrogen atom in the triazolic ring and the iron atom in the protoporphyrin moiety. The remainder of the molecule apart from the triazole ring determines the manner in which a particular azole binds to the apoprotein and is also responsible for the wide variety of azole molecules [24-27]. Since the mechanism of action depends on the triazole moiety, researchers continue to seek new antifungal drugs with the triazole core, despite the mechanisms of resistance exhibited by some fungal pathogens.

The recent efforts by various research groups to elaborate 1,2,3-triazole isomers as antifungal drugs have yielded promising compounds with a good in vitro antifungal effect [28-32]. The ongoing research by our group is focused on the development of novel antifungal 1,2,3-triazoles (e.g., A, B and $\mathbf{C}$, Scheme 2) [33-35] with dual pharmacophoric derivatives. Of the two linked pharmacophores, the main one is a 1,2,3-triazole core. Since the benzylic group has been recognized as an efficient pharmacophore [36-39], a novel library of benzylic 1,2,3-triazole derivatives was presently developed and tested. Related compounds are described in the literature as having efficient fungicidal activity (e.g., $\mathbf{D}$ and $\mathbf{E}$ ) [40-41]. 


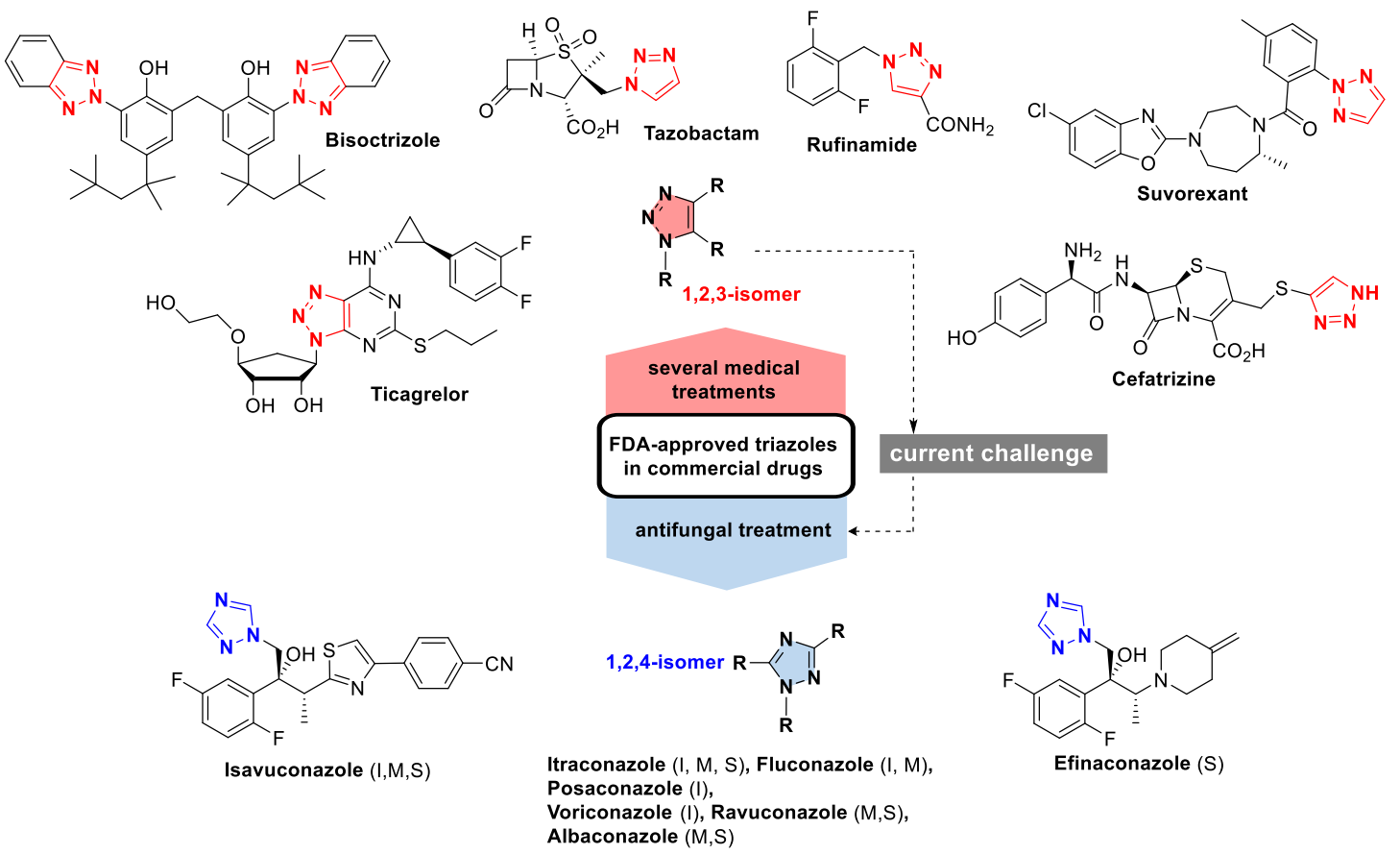

Scheme 1. Clinical drugs that are triazole derivatives, based on the 1,2,3- or 1,2,4-isomer. The1,2,4-isomer is part of the structure of some conventional pharmaceuticals used to treat invasive (I), mucosal (M), and superficial (S) fungal infections. The 1,2,3-triazole scaffold provides the basis for the antifungal drugs of choice.
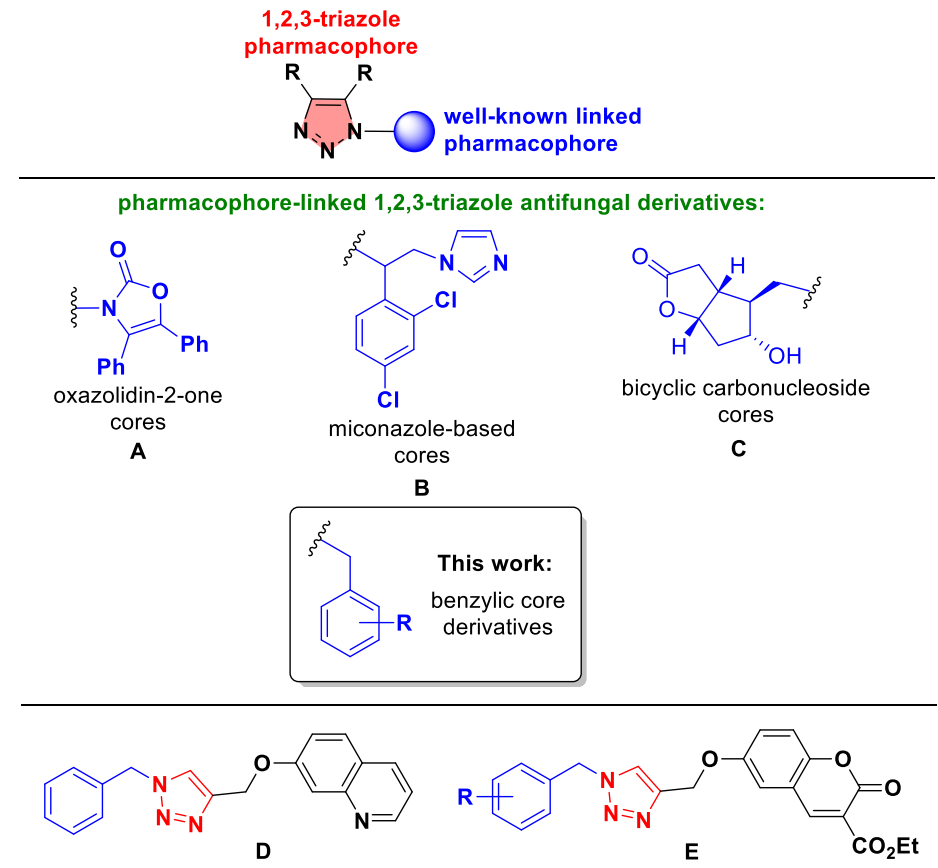

Scheme 2. Benzylic scaffolds were presently integrated into dual-pharmacophore fungicidal derivatives A, B and $\mathbf{C}$, previously developed by our group. The antifungal activity of other benzylic triazoles (e.g., $\mathbf{D}$ and $\mathbf{E}$ ) has been demonstrated. 


\section{Experimental}

\section{Chemistry}

All chemicals were of analytical grade and acquired from Merck and Sigma-Aldrich Company. Flash column chromatography was carried out by utilizing $\mathrm{SiO}_{2} 60$ (230-400 mesh). Reactions were monitored by TLC by using silica plates 60 and F254 aluminum sheets and were visualized with UV light at $254 \mathrm{~nm}$. Melting points were determined for all newly synthesized compounds in open capillary tubes on a Fischer-Johns Scientific melting point apparatus. ${ }^{1} \mathrm{H}$ and ${ }^{13} \mathrm{C}$ NMR spectra were recorded on Bruker Avance $300 \mathrm{MHz}$ and Varian $500 \mathrm{MHz}$ instruments, with $\delta$ expressed in ppm and $\mathrm{Me}_{4} \mathrm{Si}$ as the internal standard.

\section{General procedure for the synthesis of benzylic 1,2,3-triazole-4-carboxamides (3a-m)}

To a solution of $\beta$-ketonitrile $2(0.5 \mathrm{mmol})$ in t-BuOH anh. $(1.0 \mathrm{~mL})$ was added DBU $(0.6 \mathrm{mmol})$ and benzyl azide $1(0.5 \mathrm{mmol})$ under inert atmosphere. The reaction mixture was stirred at $70{ }^{\circ} \mathrm{C}$ for $24 \mathrm{~h}$. The progress of the reaction was monitored by TLC, which indicated when the starting materials disappeared. At this point, t-BuOK $(1.5 \mathrm{mmol})$ was added to the reaction mixture, followed by continuous stirring for $10 \mathrm{~h}$ at room temperature. Upon completion of this time, TLC evidenced the appearance of the corresponding 1,2,3triazole-4-carboxamide $(3)$. Brine $(\sim 30 \mathrm{~mL})$ was added and then the reaction mixture was washed with EtOAc $(3 \times 10 \mathrm{~mL})$. The organic layer was dried with $\mathrm{Na}_{2} \mathrm{SO}_{4}$ and the solvent was evaporated under reduced pressure. Flash column chromatography furnished the pure triazole.

\section{5-([1,1'-biphenyl]-4-yl)-1-(2,6-dichlorobenzyl)-1H-1,2,3-triazole-4-carboxamide (3a)}

Following the general procedure, 3a was isolated as a yellowish solid $(72 \%)$; $\mathrm{mp} 81-83^{\circ} \mathrm{C}$; Rf: 0.15 (Hex/EtOAc 70/30 X3). ${ }^{1} \mathbf{H}-\mathbf{N M R}\left(300 \mathrm{MHz} \mathrm{CDCl}_{3}\right): \delta(\mathrm{ppm}) 7.77-7.57(\mathrm{~m}, 5 \mathrm{H}), 7.49-7.35(\mathrm{~m}, 3 \mathrm{H}), 7.58$ - $7.34(\mathrm{~m}, 5 \mathrm{H}), 7.34-7.175(\mathrm{~m}, 2 \mathrm{H}), 7.11(\mathrm{~s}, 1 \mathrm{H}, \mathrm{NH}), 5.65\left(\mathrm{~s}, 2 \mathrm{H}, \mathrm{CH}_{2}\right) ;{ }^{13} \mathrm{C}-\mathbf{N M R}\left(75 \mathrm{MHz} \mathrm{CDCl}_{3}\right): \delta$ (ppm) $162.30(\mathrm{C}=\mathrm{O}), 142.86,140.19,139.78,137.85,136.70,130.69,130.39,129.84,129.10,128.93,128.53$, 127.89, 127.70, 127.30, 127.22, 124.60, 48.12 (CH $)$.; EIMS m/z 422 (23), 221 (11), 159 (36), 43 (100).

\section{5-([1,1'-biphenyl]-4-yl)-1-(3,4-bis(benzyloxy)benzyl)-1H-1,2,3-triazole-4-carboxamide (3b)}

Following the general procedure, $3 \mathbf{b}$ was provided as white crystals $(69 \%)$; mp $141-143^{\circ} \mathrm{C}$; Rf: 0.16 (Hex/EtOAc 70/30 X3). ${ }^{1} \mathbf{H}-\mathbf{N M R}\left(300 \mathrm{MHz}, \mathrm{CDCl}_{3}\right): \delta(\mathrm{ppm}) 7.64-7.56(\mathrm{~m}, 4 \mathrm{H}), 7.50-7.21$ (m, $\left.15 \mathrm{H}\right), 7.11$ $(\mathrm{s}, 1 \mathrm{H}, \mathrm{NH}), 6.78(\mathrm{~d}, \mathrm{~J}=8.2 \mathrm{~Hz}, 1 \mathrm{H}), 6.59(\mathrm{~d}, \mathrm{~J}=2.1 \mathrm{~Hz}, 1 \mathrm{H}), 6.54(\mathrm{dd}, \mathrm{J}=8.2,2.1 \mathrm{~Hz}, 1 \mathrm{H}), 5.48(\mathrm{~s}, 1 \mathrm{H}, \mathrm{NH})$, $5.35\left(\mathrm{~s}, 2 \mathrm{H}, \mathrm{CH}_{2}\right), 5.12\left(\mathrm{~s}, 2 \mathrm{H},-\mathrm{O}-\mathrm{CH}_{2}\right), 5.01\left(\mathrm{~s}, 2 \mathrm{H},-\mathrm{O}-\mathrm{CH}_{2}\right)$; ${ }^{13} \mathbf{C}-\mathbf{N M R}\left(75 \mathrm{MHz}, \mathrm{CDCl}_{3}\right) \delta(\mathrm{ppm})$ : 162.03(C=O), 148.92, 142.74, 140.03, 139.31, 138.45, 136.95, 136.73, 130.40, 128.94, 128.51, 128.49, 127.93, $127.89,127.72,127.22,127.19,127.15,124.62,120.84,114.85,114.24,71.15\left(\mathrm{O}-\mathrm{CH}_{2}\right), 71.01\left(\mathrm{O}-\mathrm{CH}_{2}\right), 51.94$ $\left(\mathrm{CH}_{2}\right)$; EIMS m/z 566 (10), 355 (8), 212 (10), 91 (100).

\section{5-([1,1'-biphenyl]-4-yl)-1-(2,3-dimethoxybenzyl)-1H-1,2,3-triazole-4-carboxamide (3c)}

Following the general procedure, 3c was afforded as yellowish crystals (68\%); mp $142-145^{\circ} \mathrm{C}$; $\mathrm{Rf}$ : $0.15(\mathrm{Hex} / \mathrm{EtOAc} 70 / 30 \mathrm{X} 3) .{ }^{\mathbf{1}} \mathbf{H}-\mathbf{N M R}\left(300 \mathrm{MHz}, \mathrm{CDCl}_{3}\right): \delta(\mathrm{ppm}) 7.67-7.57(\mathrm{~m}, 4 \mathrm{H}), 7.50-7.33(\mathrm{~m}, 5 \mathrm{H})$, $6.97(\mathrm{t}, \mathrm{J}=8.0 \mathrm{~Hz}, 1 \mathrm{H}), 6.86(\mathrm{dd}, \mathrm{J}=8.0,1.6 \mathrm{~Hz}, 1 \mathrm{H}), 6.47(\mathrm{dd}, \mathrm{J}=7.9,1.5 \mathrm{~Hz}, 1 \mathrm{H}), 5.65(\mathrm{~s}, 1 \mathrm{H}, \mathrm{NH}), 5.51(\mathrm{~s}$, $\left.2 \mathrm{H}, \mathrm{CH}_{2}\right), 3.83(\mathrm{~s}, 3 \mathrm{H}, \mathrm{OMe}), 3.61(\mathrm{~s}, 3 \mathrm{H}, \mathrm{OMe})$; ${ }^{13} \mathrm{C}-\mathrm{NMR}\left(75 \mathrm{MHz}, \mathrm{CDCl}_{3}\right): \delta(\mathrm{ppm}) 162.39(\mathrm{C}=\mathrm{O}), 152.58$, $146.31,142.80,140.22,139.80,138.22,137.88,130.48,129.51,129.05,128.24,127.86,127.23,125.32$, 124.65, 124.26, 120.13, 112.72, 60.41(OMe), $55.78(\mathrm{OMe}), 47.27\left(\mathrm{CH}_{2}\right)$.; EIMS m/z 414 (80), $221(8), 151$ (50), $136(90), 91(100)$.

\section{1-(2,6-dichlorobenzyl)-5-phenyl-1H-1,2,3-triazole-4-carboxamide (3d)}

The general procedure gave $\mathbf{3 d}$ as a yellowish powder $(65 \%)$; mp 200-201 ${ }^{\circ} \mathrm{C}$; Rf: 0.15 (Hex/EtOAc 70/30 X3). ${ }^{1} \mathbf{H}-\mathbf{N M R}\left(300 \mathrm{MHz} \mathrm{CDCl}_{3}\right): \delta(\mathrm{ppm}) 7.59-7.38(\mathrm{~m}, 5 \mathrm{H}), 7.33-7.17(\mathrm{~m}, 3 \mathrm{H}), 7.07(\mathrm{~s}, 1 \mathrm{H}, \mathrm{NH})$, $5.62(\mathrm{~s}, 1 \mathrm{H}, \mathrm{NH}), 5.59$ (s, 2H).; ${ }^{13} \mathrm{C}-\mathrm{NMR}\left(75 \mathrm{MHz}, \mathrm{CDCl}_{3}\right): \delta(\mathrm{ppm}) 160.32(\mathrm{C}=\mathrm{O}), 138.10,136.00,134.86$, 128.85, 128.22, 128.12, 127.99, 126.75, 126.68, 124.02, $46.15\left(\mathrm{CH}_{2}\right)$.; EIMS m/z 346 (10), 311 (34), 187 (3), 159 (100), 89 (48). 


\section{1-(benzo[d][1,3]dioxol-5-ylmethyl)-5-phenyl-1H-1,2,3-triazole-4-carboxamide (3e)}

Following the general procedure, $3 \mathbf{e}$ was produced as a white powder $(75 \%) ; \mathrm{mp} 170-173^{\circ} \mathrm{C}$; Rf: 0.14 (Hex/EtOAc 70/30 X3). ${ }^{1} \mathbf{H}-N M R\left(300 \mathrm{MHz}, \mathrm{DMSO}_{\mathrm{d}}\right.$ ): $\delta(\mathrm{ppm}) 7.83$ (s, 1H, NH), $7.62-7.40(\mathrm{~m}, 3 \mathrm{H}), 7.43$ - $7.32(\mathrm{~m}, 2 \mathrm{H}), 6.78(\mathrm{~d}, \mathrm{~J}=8.0 \mathrm{~Hz}, 1 \mathrm{H}), 6.48(\mathrm{~d}, \mathrm{~J}=1.7 \mathrm{~Hz}, 1 \mathrm{H}), 6.37(\mathrm{dd}, \mathrm{J}=8.0,1.8 \mathrm{~Hz}, 1 \mathrm{H}), 5.97(\mathrm{~s}, 2 \mathrm{H}$, $\left.\mathrm{OCH}_{2} \mathrm{O}-\right), 5.39\left(\mathrm{~s}, 2 \mathrm{H}, \mathrm{CH}_{2}\right) . ;{ }^{13} \mathrm{C}-\mathrm{NMR}\left(75 \mathrm{MHz}, \mathrm{DMSO}-\mathrm{d}_{6}\right): \delta(\mathrm{ppm}) 162.24(\mathrm{C}=\mathrm{O}), 147.86,147.41,139.57$, $138.90,130.47,130.01,129.36,128.76,126.67,121.43,108.71,108.19,101.64\left(-\mathrm{OCH}_{2} \mathrm{O}-\right), 51.54\left(\mathrm{CH}_{2}\right)$.; EIMS m/z 322 (25), 293 (25), 149 (20), 135 (100), 77 (60).

\section{1-(3,4-bis(benzyloxy)benzyl)-5-phenyl-1H-1,2,3-triazole-4-carboxamide (3f)}

Following the general procedure, $\mathbf{3 f}$ was furnished as a yellowish powder $(69 \%)$; mp $111-114^{\circ} \mathrm{C}$; Rf: $0.14(\mathrm{Hex} / \mathrm{EtOAc} 70 / 30 \mathrm{X} 3) .{ }^{1} \mathbf{H}-\mathrm{NMR}\left(300 \mathrm{MHz}, \mathrm{DMSO}-\mathrm{d}_{6}\right): \delta(\mathrm{ppm}) 7.84(\mathrm{~s}, 1 \mathrm{H}, \mathrm{NH}), 7.55-7.25$ (m, 15H), $6.93(\mathrm{~d}, \mathrm{~J}=8.3 \mathrm{~Hz}, 1 \mathrm{H}), 6.55(\mathrm{~d}, \mathrm{~J}=2.0 \mathrm{~Hz}, 1 \mathrm{H}), 6.47(\mathrm{dd}, \mathrm{J}=8.3,2.0 \mathrm{~Hz}, 1 \mathrm{H}), 5.38\left(\mathrm{~s}, 2 \mathrm{H}, \mathrm{CH}_{2}\right), 5.07(\mathrm{~s}, 2 \mathrm{H}$,

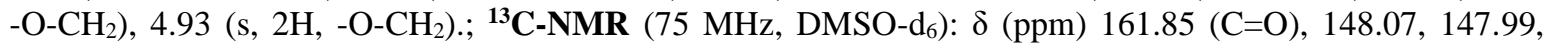
$139.15,138.44,137.12,136.93,130.08,129.53,128.46,128.43,128.32,128.09,127.90,127.85,127.68$, $127.56,126.37,120.37,114.40,113.73,70.07\left(\mathrm{O}-\mathrm{CH}_{2}\right), 51.12\left(\mathrm{CH}_{2}\right)$.

\section{1-(2,3-dimethoxybenzyl)-5-phenyl-1H-1,2,3-triazole-4-carboxamide (3g)}

The general procedure led to $\mathbf{3 g}$ as a white powder (71\%); mp 206-208 ${ }^{\circ} \mathrm{C}$; Rf: 0.15 (Hex/EtOAc 70/30 X3). ${ }^{1}$ H-NMR (300 MHz, DMSO-d 6 ): $\delta(\mathrm{ppm}) 7.66(\mathrm{~s}, 1 \mathrm{H}, \mathrm{NH}), 7.52-7.34(\mathrm{~m}, 5 \mathrm{H}), 7.32(\mathrm{~s}, 1 \mathrm{H}, \mathrm{NH}), 7.02$ - $6.90(\mathrm{~m}, 2 \mathrm{H}), 6.39(\mathrm{dd}, \mathrm{J}=5.5,3.7 \mathrm{~Hz}, 1 \mathrm{H}), 5.42\left(\mathrm{~s}, 2 \mathrm{H}, \mathrm{CH}_{2}\right), 3.79(\mathrm{~s}, 3 \mathrm{H}, \mathrm{OMe}), 3.46(\mathrm{~s}, 3 \mathrm{H}, \mathrm{OMe}) . ;{ }^{13} \mathrm{C}-$ NMR (125 MHz, $\left.\mathrm{CDCl}_{3} / \mathrm{DMSO}_{-} \mathrm{d}_{6}\right): \delta(\mathrm{ppm}) 162.19(\mathrm{C}=\mathrm{O}), 152.54,146.24,139.12,130.36,129.74,128.99$, 128.49, 126.69, 124.30, 120.37, 113.15, $60.00(\mathrm{OMe}), 55.98(\mathrm{OMe}), 47.07\left(\mathrm{CH}_{2}\right) . ;$ EIMS m/z 338 (75), 307 (62), 136 (91), 91 (100).

\section{1-(4-methoxybenzyl)-5-phenyl-1H-1,2,3-triazole-4-carboxamide (3h)}

The general procedure resulted in $\mathbf{3 h}$ as a white powder (70\%); $\mathrm{mp} 209-210^{\circ} \mathrm{C}$; Rf: 0.15 (Hex/EtOAc 70/30 X3). ${ }^{1} \mathbf{H}-N M R\left(300 \mathrm{MHz}, \mathrm{CDCl}_{3}\right): \delta(\mathrm{ppm}) 7.56-7.38(\mathrm{~m}, 3 \mathrm{H}), 7.34-7.23(\mathrm{~m}, 2 \mathrm{H}), 7.08(\mathrm{~s}, 1 \mathrm{H}, \mathrm{NH})$, $6.95(\mathrm{~d}, \mathrm{~J}=8.2 \mathrm{~Hz}, 2 \mathrm{H}), 6.78(\mathrm{~d}, \mathrm{~J}=8.1 \mathrm{~Hz}, 2 \mathrm{H}), 5.47(\mathrm{~s}, 1 \mathrm{H}, \mathrm{NH}), 5.36\left(\mathrm{~s}, 2 \mathrm{H}, \mathrm{CH}_{2}\right), 3.77$ (s, 3H, OMe).; ${ }^{13} \mathrm{C}-$ NMR $\left(125 \mathrm{MHz}, \mathrm{CDCl}_{3}\right): \delta(\mathrm{ppm}) 162.01(\mathrm{C}=\mathrm{O}), 159.60,139.49,138.38,129.97,129.08,128.55,128.50$, 126.69, 125.90, 114.15, 55.27 (OMe), $51.65\left(\mathrm{CH}_{2}\right)$.; EIMS m/z 308 (60), 279 (55), 121 (100), 77 (40).

\section{1-(2,6-dichlorobenzyl)-5-(p-tolyl)-1H-1,2,3-triazole-4-carboxamide (3i)}

Following the general procedure, $\mathbf{3 i}$ was obtained as yellowish crystals; yield $(76 \%)$; mp $187-189^{\circ} \mathrm{C}$; Rf: 0.14 (Hex/EtOAc 70/30 X3). ${ }^{1} \mathbf{H}-\mathbf{N M R}\left(300 \mathrm{MHz}, \mathrm{CDCl}_{3}\right): \delta(\mathrm{ppm}) 7.38(\mathrm{~d}, \mathrm{~J}=8.2 \mathrm{~Hz}, 2 \mathrm{H}), 7.34-7.25$ $(\mathrm{m}, 4 \mathrm{H}), 7.22(\mathrm{dd}, \mathrm{J}=9.2,6.7 \mathrm{~Hz}, 1 \mathrm{H}), 7.07(\mathrm{~s}, 1 \mathrm{H}, \mathrm{NH}), 5.69(\mathrm{~s}, 1 \mathrm{H}, \mathrm{NH}), 5.57\left(\mathrm{~s}, 2 \mathrm{H}, \mathrm{CH}_{2}\right), 2.42(\mathrm{~s}, 3 \mathrm{H}$, $\left.\mathrm{CH}_{3}\right)$.; ${ }^{13} \mathrm{C}-\mathrm{NMR}\left(75 \mathrm{MHz}, \mathrm{CDCl}_{3}\right): \delta(\mathrm{ppm}) \delta 162.29(\mathrm{C}=\mathrm{O}), 140.16,139.99,137.67,136.64,130.58,129.90$, 129.79, 129.29, 128.43, 122.72, $47.80\left(\mathrm{CH}_{2}\right), 21.44\left(\mathrm{CH}_{3}\right)$.; EIMS m/z 361 (19), 325 (30), 159 (100), 77 (21).

\section{1-(benzo[d][1,3]dioxol-5-ylmethyl)-5-(p-tolyl)-1H-1,2,3-triazole-4-carboxamide (3j)}

Following the general procedure, $\mathbf{3 j}$ was formed as yellow crystals $(76 \%)$;p $157-159^{\circ} \mathrm{C}$; Rf: 0.15 (Hex/EtOAc 70/30 X3). ${ }^{\mathbf{1}} \mathbf{H}-\mathbf{N M R}\left(300 \mathrm{MHz}, \mathrm{CDCl}_{3}\right): \delta(\mathrm{ppm}) 7.26(\mathrm{~d}, \mathrm{~J}=8.2 \mathrm{~Hz}, 2 \mathrm{H}), 7.19(\mathrm{~d}, \mathrm{~J}=8.2 \mathrm{~Hz}$, 2H), $7.08(\mathrm{~s}, 1 \mathrm{H}, \mathrm{NH}), 6.68(\mathrm{~d}, \mathrm{~J}=8.0 \mathrm{~Hz}, 1 \mathrm{H}), 6.57(\mathrm{~d}, \mathrm{~J}=1.8 \mathrm{~Hz}, 1 \mathrm{H}), 6.48(\mathrm{dd}, \mathrm{J}=8.0,1.8 \mathrm{~Hz}, 1 \mathrm{H}), 5.93(\mathrm{~s}$, 2H, -OCH2O-), $5.64(\mathrm{~s}, 1 \mathrm{H}, \mathrm{NH}), 5.31\left(\mathrm{~s}, 2 \mathrm{H}, \mathrm{CH}_{2}\right), 2.42\left(\mathrm{~s}, 3 \mathrm{H}, \mathrm{CH}_{3}\right) .{ }^{13} \mathbf{C}-\mathbf{N M R}\left(125 \mathrm{MHz}, \mathrm{CDCl}_{3}\right): \delta(\mathrm{ppm})$ $162.16(\mathrm{C}=\mathrm{O}), 147.99,147.65,140.19,139.63,138.28,129.79,129.30,128.42,122.66,121.32,108.29,108.11$, 101.25, $51.70\left(-\mathrm{OCH}_{2} \mathrm{O}-\right), 21.43\left(\mathrm{CH}_{3}\right)$; EIMS m/z 336 (32), 149 (12), 135 (100), 77 (56).

\section{1-(3,4-bis(benzyloxy)benzyl)-5-(p-tolyl)-1H-1,2,3-triazole-4-carboxamide (3k)}

Following the general procedure, $\mathbf{3 k}$ was prepared as colorless crystals $(73 \%) ; \mathrm{mp} 117-119^{\circ} \mathrm{C}$; Rf: 0.16 (Hex/EtOAc 70/30 X3). ${ }^{\mathbf{1}} \mathbf{H}-\mathbf{N M R}\left(300 \mathrm{MHz}, \mathrm{CDCl}_{3}\right): \delta(\mathrm{ppm}) \delta 7.46-7.24(\mathrm{~m}, 10 \mathrm{H}), 7.19(\mathrm{~d}, \mathrm{~J}=8.2 \mathrm{~Hz}$, 2H), $7.07(\mathrm{~d}, \mathrm{~J}=8.2 \mathrm{~Hz}, 3 \mathrm{H}, \mathrm{Ar}, \mathrm{NH}),, 6.78(\mathrm{~d}, \mathrm{~J}=8.2 \mathrm{~Hz}, 1 \mathrm{H}), 6.59(\mathrm{~d}, \mathrm{~J}=2.1 \mathrm{~Hz}, 1 \mathrm{H}), 6.53(\mathrm{dd}, \mathrm{J}=8.2,2.1$ $\mathrm{Hz}, 1 \mathrm{H}), 5.52(\mathrm{~s}, 1 \mathrm{H}, \mathrm{NH}), 5.29\left(\mathrm{~s}, 2 \mathrm{H}, \mathrm{CH}_{2}\right), 5.12\left(\mathrm{~s}, 2 \mathrm{H},-\mathrm{OCH}_{2}\right), 5.02\left(\mathrm{~s}, 2 \mathrm{H},-\mathrm{OCH}_{2}\right), 2.39\left(\mathrm{~s}, 3 \mathrm{H}, \mathrm{CH}_{3}\right)$; ${ }^{13}$ C-NMR (125 MHz, $\left.\mathrm{CDCl}_{3}\right): \delta(\mathrm{ppm}) 162.09(\mathrm{C}=\mathrm{O}), 148.89,148.86,140.06,139.59,138.27,136.95,136.79$, 
$129.79,129.24,128.49,128.48,127.90,127.85,127.23,127.20,122.73,120.80,114.84,114.27,71.15(-$ $\left.\mathrm{OCH}_{2}\right), 71.03\left(-\mathrm{OCH}_{2}\right), 51.69\left(\mathrm{CH}_{2}\right), 21.45\left(\mathrm{CH}_{3}\right)$; EIMS m/z 504 (12), 413 (2), 293 (11), 91 (100).

\section{1-(2,3-dimethoxybenzyl)-5-(p-tolyl)-1H-1,2,3-triazole-4-carboxamide (3I)}

The general procedure gave $3 \mathrm{l}$ as a white powder; yield $(82 \%)$; mp $185-188^{\circ} \mathrm{C}$; Rf: 0.14 (Hex/EtOAc 70/30 X3). ${ }^{1} \mathrm{H}-\mathrm{NMR}\left(300 \mathrm{MHz}, \mathrm{CDCl}_{3}\right): \delta(\mathrm{ppm}) 7.24(\mathrm{~s}, 4 \mathrm{H}), 7.12(\mathrm{~s}, 1 \mathrm{H}, \mathrm{NH}), 6.97(\mathrm{t}, \mathrm{J}=8.0 \mathrm{~Hz}, 1 \mathrm{H}), 6.88$ $(\mathrm{td}, \mathrm{J}=8.1,1.6 \mathrm{~Hz}, 1 \mathrm{H}), 6.43(\mathrm{dd}, \mathrm{J}=8.1,1.6 \mathrm{~Hz}, 1 \mathrm{H}), 5.66(\mathrm{~s}, 1 \mathrm{H}, \mathrm{NH}), 5.46\left(\mathrm{~s}, 2 \mathrm{H}, \mathrm{CH}_{2}\right), 3.84(\mathrm{~s}, 3 \mathrm{H}, \mathrm{OMe})$, 3.62 (s, 3H, OMe), 2.38 (s, 3H, $\left.\mathrm{CH}_{3}\right)$.; ${ }^{13} \mathrm{C}-\mathrm{NMR}\left(75 \mathrm{MHz}, \mathrm{CDCl}_{3}\right): \delta(\mathrm{ppm}) 162.29(\mathrm{C}=\mathrm{O}), 152.45,146.14$, $140.02,140.00,138.04,129.77,129.20,128.83,124.17,122.67,119.95,112.52,60.20(\mathrm{OMe}), 55.71(\mathrm{OMe})$, $46.96\left(\mathrm{CH}_{2}\right), 21.37\left(\mathrm{CH}_{3}\right)$.; EIMS m/z 352 (55), 321 (58), 136 (100), 92 (81).

\section{1-(4-methoxybenzyl)-5-(p-tolyl)-1H-1,2,3-triazole-4-carboxamide (3m)}

The general procedure produced $\mathbf{3 m}$ as a white powder; yield $(69 \%) ; \mathrm{mp} 190-191^{\circ} \mathrm{C}$; Rf: 0.15 (Hex/EtOAc 70/30 X3). ${ }^{1} \mathbf{H}-N M R\left(300 \mathrm{MHz}, \mathrm{CDCl}_{3}\right): \delta(\mathrm{ppm}) 7.26(\mathrm{~d}, \mathrm{~J}=8.2 \mathrm{~Hz}, 2 \mathrm{H}), 7.17(\mathrm{~d}, \mathrm{~J}=8.2 \mathrm{~Hz}$, $2 \mathrm{H}), 7.07(\mathrm{~s}, 1 \mathrm{H}, \mathrm{NH}), 7.02-6.96(\mathrm{~m}, 2 \mathrm{H}), 6.82-6.76(\mathrm{~m}, 2 \mathrm{H}), 5.56(\mathrm{~s}, 1 \mathrm{H}, \mathrm{NH}), 5.35\left(\mathrm{~s}, 2 \mathrm{H}, \mathrm{CH}_{2}\right), 3.77(\mathrm{~s}$, $3 \mathrm{H}, \mathrm{OMe}), 2.42\left(\mathrm{~s}, 3 \mathrm{H}, \mathrm{CH}_{3}\right) \cdot{ }^{13} \mathrm{C}-\mathrm{NMR}\left(75 \mathrm{MHz}, \mathrm{CDCl}_{3}\right): \delta(\mathrm{ppm}) 162.15(\mathrm{C}=\mathrm{O}), 159.56,140.12,139.60$, 138.27, 129.83, 129.28, 129.04, 126.87, 122.76, 114.12, $55.25(\mathrm{OMe}), 51.47\left(\mathrm{CH}_{2}\right), 21.46\left(\mathrm{CH}_{3}\right)$.; EIMS m/z 322 (25), 136 (35), 121 (100), 91 (40).

\section{Fungicidal activity}

The antifungal impact of 3a-3m was analyzed in vitro with the microdilution techniques for testing antimicrobial susceptibility. All assays were performed in triplicate. The M38-A2 method was employed to examine activity against filamentous fungi [42-44], while the M27-A3 method described by the CLSI was adopted to assess the effects against yeasts [45-47].

The filamentous fungal strains consisted of M. hiemalis ATCC-8690, A. fumigatus ATCC-16907, T. cutaneum ATCC-28592 and R. oryzae ATCC-10329. The yeast specimens were comprised of C. albicans ATCC-10231, C. utilis ATCC-9226, C. krusei ATCC-14243 and C. glabrata ATCC-34138.

For antifungal evaluations, strains of clinical importance in Mexico, Latin America and Europe were considered. Candidiasis is the most frequent superficial and systemic mycosis in neonates, immunocompromised individuals, and hospitalized patients [48]. One strain of filamentous fungi from each of the genera Rhizopus, Aspergillus and Mucor can cause systemic infections and severe gastrointestinal disorders [49]. The fungi were cultured in RPMI 1640 synthetic medium containing glutamine but not sodium bicarbonate, a morpholino propane sulfonic acid (MOPS) buffer at $0.164 \mathrm{M}$ adjusted to $\mathrm{pH} 7 \pm 0.1$, and $0.2 \%$ glucose.

Because the synthesized compounds are not soluble in water, itraconazole served as the standard drug in the exploration of antimicrobial sensitivity. Based on the CLSI methodology that establishes the microdilutions for the reference drug, a solution of itraconazole at $1600 \mu \mathrm{g} / \mathrm{ml}$ was dissolved in dimethyl sulfoxide to reach concentrations ranging from $16 \mu \mathrm{g} / \mathrm{mL}$ to $0.03 \mu \mathrm{g} / \mathrm{mL}$, and the corresponding values in $\mathrm{mmol} / \mathrm{mL}$ were determined and utilized for the solutions of $\mathbf{3 a - 3 m}$ (Table 1).

In accordance with the CLSI, the minimum inhibitory concentrations (MIC) of the compounds and the standard drug were determined with the help of an inverted mirror, performing all experiments in triplicate. The different concentrations of the test compounds and reference drug were added to 96 -well plates containing RPMI 1640 medium buffered with MOPS (3-[N-morpholino] propane sulfonic acid) (Sigma-Aldrich). MIC values are expressed in mmol per milliliter.

After $24 \mathrm{~h}$ growth on SDA plates, 3-5 fungal colonies $\geq 1 \mathrm{~mm}$ were gathered with a culture loop, resuspended in saline solution $(0.85 \% \mathrm{NaCl})$, stirred well and adjusted to $0.5 \mathrm{McFarland}$ optical density with the aid of a spectrophotometer (wavelength $530 \mathrm{~nm}$ ). This solution, having a concentration of $1-5 \times 10^{6} \mathrm{CFU} / \mathrm{ml}$, was diluted (1:1000) with RPMI medium and utilized to inoculate the plates containing the antifungal compounds. The wells of columns 2-11 were inoculated with $100 \mu \mathrm{l}$ of the yeast suspension. Column 1 had 200 $\mu l$ of RPMI in each well and did not receive inoculation, thus serving as the sterility control of the medium. The wells in column 12 were inoculated but did not contain any antifungal compound, serving as the growth control. For all strains of yeast and filamentous fungi, the plates were incubated at $35^{\circ} \mathrm{C}$ for $48 \mathrm{~h}$. 
The visual reading of fungal growth was carried out with an inverted mirror. For the azoles, the MIC is the lowest concentration of an antifungal that produces a substantial reduction in yeast growth $(\geq 50 \%)$ and a $100 \%$ inhibition of the growth of filamentous fungi, in each case compared to the corresponding control.

\section{Results and discussion}

\section{Chemistry}

Our group recently reported a novel two-step methodology for synthesizing benzylic 1,2,3-triazole-4carboxamide derivatives 3 [50]. The first step is a highly regioselective azide-enolate 1,3-dipolar cycloaddition (coupling benzyl azide $1 / \beta$-ketonitrile 2 ) in dimethylformamide to generate the benzylic 1,2,3-triazole-4carbonitrile adducts 4 , followed by their isolation and identification. The second step is a hydrolysis of the nitrile group to afford triazolic compounds 3 as antifungal agents.

A library of novel benzylic 1,2,3-triazole-4-carboxamide derivatives was presently designed and then elaborated via an optimized protocol involving a one-pot reaction that facilitates the in situ hydrolysis of the nitrile (Scheme 3). Thirteen novel benzylic 1,2,3-triazole-4-carboxamides (3a-m) were obtained with acceptable yields $(65-82 \%)$. For the current synthetic procedure, the solvent was tert-butanol rather than dimethylformamide. The latter, included in the previous two-step protocol, had the drawback of remaining in the reaction products during the purification process. Hence, the one-step methodology is eco-friendly. For the 1,3-dipolar cycloaddition (promoted by DBU), the temperature was $70^{\circ} \mathrm{C}$ and the reaction time $24 \mathrm{~h}$. For the hydrolysis, the second part of the one-pot reaction was carried out at room temperature for $10 \mathrm{~h}$. Anhydrous $t$ $\mathrm{BuOH}$ proved to be an excellent solvent.

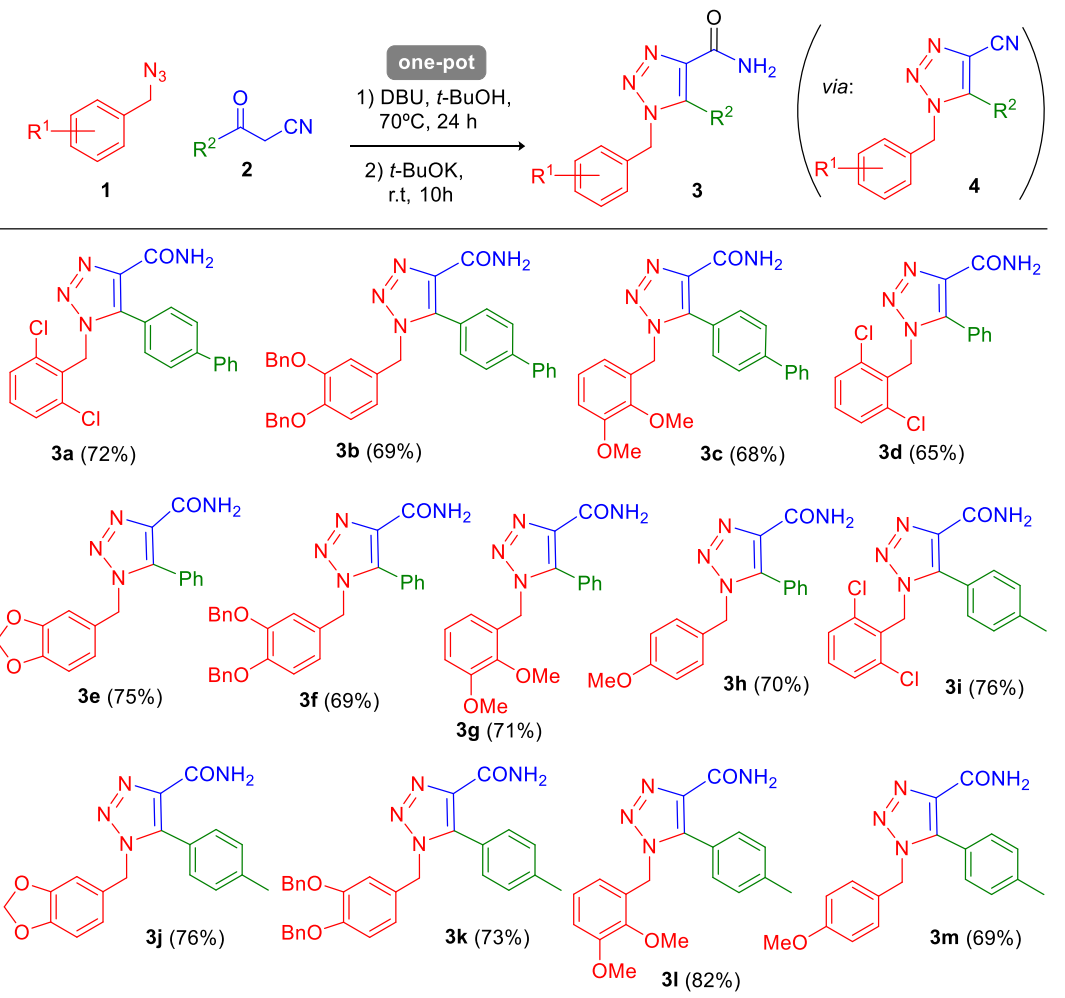

Scheme 3. One-pot synthesis of 1,2,3-triazole-4-carboxamides 3a-m via hydrolysis of 5-nitrile substituted triazolic intermediates (4). The latter were afforded by azide-enolate cycloaddition (pericyclic coupling between azides 1 and $\beta$-ketonitriles 2). Reaction conditions: $\beta$-ketonitrile 2 (1.0 eq), benzyl azide 1 (1.0 eq), and DBU (1.1 eq) in anh. $t$ - $\mathrm{BuOH}$ under inert atmosphere was stirred at $70{ }^{\circ} \mathrm{C}$ for $24 \mathrm{~h}$. Then $t$-BuOK (3.0 eq) was added, and the reaction mixture was stirred at $\mathrm{rt}$ for $10 \mathrm{~h}$. 


\section{Fungicidal activity}

According to the microdilution techniques of antifungal susceptibility testing protocols (CLSI), itraconazole was prepared at ten concentrations from $16 \mu \mathrm{g} / \mathrm{mL}$ to $0.03 \mu \mathrm{g} / \mathrm{mL}$, as shown in Table 1 . The corresponding values in $\mathrm{mmol} / \mathrm{mL}$ were determined and utilized for the solutions of $\mathbf{3 a - 3} \mathbf{m}$.

Table 1. Concentrations of itraconazole used to assess antifungal sensitivity, following the protocol of the CLSI.

\begin{tabular}{|l|l|l|l|l|l|l|l|l|l|l|}
\hline CLSI $\boldsymbol{\mu g} / \mathbf{m L}$ & 16 & 8 & 4 & 2 & 1 & 0.5 & 0.25 & 0.12 & 0.06 & 0.03 \\
\hline $\boldsymbol{\mu m o l} / \mathbf{m L}$ & 2.6 & 1.13 & 0.56 & 0.28 & 0.14 & 0.07 & 0.035 & 0.017 & 0.008 & 0.004 \\
\hline
\end{tabular}

The outcome of the in vitro antifungal evaluation of $\mathbf{3 a - 3} \mathbf{m}$ and itraconazole is expressed as the mean of the MIC of three assays (Table 2), found in relation to the filamentous fungi (M. hiemalis ATCC-8690, A. fumigatus ATCC-16907, T. cutaneum ATCC-28592 and R. oryzae ATCC-10329) and the yeasts (C. albicans ATCC-10231, C. utilis ATCC-9226, C. krusei ATCC-14243 and C. glabrata ATCC-34138).

Table 2. The in vitro antifungal activity of the synthesized compounds is expressed as the MIC $(\mu \mathrm{mol} / \mathrm{mL})$.

\begin{tabular}{|c|c|c|c|c|c|c|c|c|}
\hline & \multicolumn{4}{|c|}{ Yeasts } & \multicolumn{4}{|c|}{ Filamentous fungi } \\
\hline Compound & C. alb. & C. uti. & C. kru. & C. gla. & M. hie. & A. fum. & T. cut. & R. ory. \\
\hline $\mathbf{3 a}$ & 2.6 & 2.6 & 2.6 & 2.6 & 2.6 & 2.6 & 2.6 & 0.14 \\
\hline $3 \mathbf{b}$ & 2.6 & 2.6 & 2.6 & 2.6 & 2.6 & 2.6 & 2.6 & 0.14 \\
\hline $3 c$ & 2.6 & 2.6 & 2.6 & 2.6 & 2.6 & 2.6 & 2.6 & 0.07 \\
\hline $3 d$ & 2.6 & 2.6 & 2.6 & 2.6 & 2.6 & 2.6 & 2.6 & 0.017 \\
\hline $3 e$ & 2.6 & 2.6 & 2.6 & 2.6 & 2.6 & 2.6 & 2.6 & 0.017 \\
\hline $3 f$ & 2.6 & 2.6 & 2.6 & 2.6 & 2.6 & 2.6 & 2.6 & 2.6 \\
\hline $3 g$ & 2.6 & 2.6 & 2.6 & 2.6 & 2.6 & 2.6 & 2.6 & 2.6 \\
\hline $3 h$ & 2.6 & 2.6 & 2.6 & 2.6 & 2.6 & 2.6 & 2.6 & 1.13 \\
\hline $3 \mathbf{i}$ & 2.6 & 2.6 & 2.6 & 2.6 & 2.6 & 1.13 & 2.6 & 1.13 \\
\hline $3 \mathbf{j}$ & 2.6 & 2.6 & 2.6 & 2.6 & 2.6 & 1.13 & 2.6 & 2.6 \\
\hline $3 \mathbf{k}$ & 2.6 & 2.6 & 2.6 & 2.6 & 2.6 & 2.6 & 2.6 & 2.6 \\
\hline 31 & 2.6 & 2.6 & 2.6 & 2.6 & 2.6 & 2.6 & 2.6 & 2.6 \\
\hline $3 \mathrm{~m}$ & 2.6 & 2.6 & 2.6 & 2.6 & 2.6 & 2.6 & 2.6 & 2.6 \\
\hline Standard $^{a}$ & 0.004 & 0.035 & 0.035 & 0.14 & 0.56 & 0.14 & 1.13 & 0.14 \\
\hline
\end{tabular}

Abbreviations: C. alb., Candida albicans; C. uti., Candida utilis; C. kru., Candida krusei; C. gla., Candida glabrata; M. hie, Mucor hiemalis; A. fum, Aspergillus fumigatus; T. cut, Trichosporon cutaneum; R. ory, Rhizopus oryzae; ${ }^{a}$ Itraconazole.

Previous studies have demonstrated that certain triazoles exhibit in vitro activity against several human pathogenic fungi, including Candida species and filamentous fungi such as Aspergillus spp., Mucor spp. and Rhizopus spp. [33]. In the current contribution, thirteen synthesized compounds were tested in vitro against four filamentous fungi and four yeast specimens.

The inhibition of $R$. oryzae was excellent for $\mathbf{3 d}$ and $\mathbf{3 e}$ and good for $\mathbf{3} \mathbf{c}$, in the three cases better than the results obtained with the reference drug, itraconazole $(\mathbf{3 d}$ and $\mathbf{3 e}, \mathrm{MIC}=0.017 \mu \mathrm{mol} / \mathrm{mL} ; \mathbf{3 c}, \mathrm{MIC}=0.07$ $\mu \mathrm{mol} / \mathrm{mL}$; itraconazole, $\mathrm{MIC}=0.14 \mu \mathrm{mol} / \mathrm{L})$. Compounds $\mathbf{3 a}$ and $\mathbf{3 b}$ displayed an antifungal effect on $R$. oryzae equivalent to that of itraconazole.

None of the test compounds were active against the yeast strains or two of the filamentous fungi, $M$. hiemalis and T. cutaneum. The treatment with $\mathbf{3 i}$ and $\mathbf{3 j}$ led to a very limited impact on A. fumigatus. No structure-activity correlation was found for the yeast growth inhibition of $C$. albicans induced by the active compounds. 
For both 3d and 3e (the most efficient fungicidal agents against $R$. oryzae), the structure-activity relation pointed to the 4-phenyl-4-carboxamide triazole moiety as being responsible for the antifungal effect, while neither the electron-poor ring (substituted with 2,6-dichloro to form 3d) or electron-rich ring (containing piperonyl in 3e) were relevant. Small ethers as substituents improved the results obtained, as evidenced by the data for $\mathbf{3 c}$ and $\mathbf{3 e}$. Another critical aspect is the size of the structure. 3a, 3d and $\mathbf{3 e}$ are the smallest of the series of synthesized compounds and constitute three of the five molecules with the lowest MIC values for activity against $R$. oryzae.

The compounds that showed less activity than the reference drug bear the largest functional groups in their structure, suggesting the importance of a small substituent in positions 1 and 5 of the triazole to favor interaction with the active site of the fungus.

The antifungal effect of $\mathbf{3 d}$ and $\mathbf{3 e}$ in $R$. oryzae is of great value because this fungus produces infections in immunocompetent patients that can lead to mucormycosis [51-52]. Furthermore, since $R$. oryzae had showed resistance against 1,2,4-triazolic isomer commercial drugs [22-23], these outcomes represent an excellent opportunity for 1,2,3-triazolic isomers as serious candidates in the treatment of such infections.

\section{Conclusion}

Thirteen benzylic 1,2,3-triazole-4-carboxamides 3a-m were elaborated by a novel one-pot procedure, avoiding previous purifications and the expenditure of organic solvents. Thus, the synthetic protocol is ecofriendly. In addition, the raw materials for the reaction are economical and allow for an easy synthesis in the lab.

Based on in vitro microdilution techniques for the evaluation of antimicrobial susceptibility, $3 \mathrm{~d}$ and $3 \mathrm{e}$ proved to be the most efficient fungicidal agents against $R$. oryzae, showing greater antifungal activity than the other test compounds and the reference drug, itraconazole. Since $R$. oryzae had showed resistance against 1,2,4triazolic isomer commercial drugs, $3 \mathrm{~d}$ and $3 \mathrm{e}$ are 1,2,3-triazolic candidates for future complementary biological studies in order to increase the antifungal effect by optimizing these two new scaffolds. Future research could possibly involve the optimized compounds in clinical trials to treat clinical infections produced by Rhizopus spp. strains, especially mucormycosis in immunocompetent patients.

\section{Acknowledgements}

The authors are grateful to the Secretaría de Investigación y Estudios Avanzados/UAEMéx for the financial support provided (grant 5011/2020CIB) and to CONACyT-Mexico for postgraduate scholarships. We would like to thank the referees for their valuable comments and suggestions, and M. N. Zavala-Segovia and L. Triana-Cruz (CCIQS UAEM-UNAM) for technical support. We are beholden to Dr. David Corona-Becerril for his support in this work and acknowledge Prof. Bruce Allan Larsen for proofreading the manuscript.

\section{References}

1. Rodrigues, M. L.; Nosanchuk, J. D. PLoS Negl. Trop. Dis. 2020, 14, e0007964. https://doi.org/10.1371/journal.pntd.0007964

2. Köhler, J. R.; Casadevall, A.; Perfect, J. Cold Spring Harb. Perspect. Med. 2015, 5, a019273. https://doi.org/10.1101/cshperspect.a019273

3. Köhler, J. R.; Hube, B.; Puccia, R.; Casadevall, A.; Perfect, J. R., Chapter 39: Fungi that Infect Humans. In: The Fungal Kingdom. Heitman, J.; Howlett, B. J.; Crous, P. W.; Stukenbrock, E. H.; 
James, T. Y.; Gow, N. A. R. (Ed)., American Society for Microbiology 2017, e-ISBN: 9781555819583. https://doi.org/10.1128/microbiolspec.FUNK-0014-2016

4. Dheer, D.; Singh, V.; Shankar, R. Bioorg. Chem. 2017, 71, 30-54. https://doi.org/10.1016/j.bioorg.2017.01.010

5. Kharb, R.; Sharma, P. C.; Yar, M. S. J. Enzym. Inhib. Med. Chem. 2011, 26, 1-21. https://doi.org/10.3109/14756360903524304

6. de Carvalho da Silva, F.; Cardoso, M. F. C.; Ferreira, P. G.; Ferreira V. F. Biological Properties of 1H-1,2,3- and 2H-1,2,3-Triazoles. In: Dehaen W., Bakulev V. (eds) Chemistry of 1,2,3-triazoles. Topics in Heterocyclic Chemistry, vol 40, Springer, Cham, 2014. Online ISBN 978-3-319-07962-2. https://doi.org/10.1007/7081_2014_124

7. Massarotti, A.; Aprile, S.; Mercalli, V.; Del Grosso, E.; Grosa, G.; Sorba, G.; Tron, G. C. Chem. Med. Chem. 2014, 9, 2497-2508. https://doi.org/10.1002/cmdc.201402233

8. Agalave, S. G.; Maujan, S. R.; Pore, V. S., Chem. Asian J. 2011, 6, 2696-2718. https://doi.org/10.1002/asia.201100432

9. Howard, K. C.; Dennis, E. K.; Watt, D. S.; Garneau-Tsodikova, S. A. Chem. Soc. Rev. 2020, 49, 24262480. https://doi.org/10.1039/c9cs00556k

10. Rani, A.; Singh, G.; Singh, A.; Maqbool, U.; Kaur, G.; Singh, J. RSC Adv. 2020, 10, 5610-5635. https://doi.org/10.1039/C9RA09510A

11. Lass-Flörl, C. Drugs 2011, 71, 2405-2419. https://doi.org/10.2165/11596540-000000000-00000

12. Nett, J. E.; Andes, D. R. Infect. Dis. Clin. North Am. 2016, 30, 51-83. https://doi.org/10.1016/j.idc.2015.10.012

13. Miceli, M. H.; Kauffman, C. A. Clin. Infect. Dis. 2015, 61, 1558-1565. https://doi.org/10.1093/cid/civ571

14. Chang, Y. L.; Yu, S. J.; Heitman, J.; Wellington, M.; Chen, Y. L. Virulence 2017, 8, 222-236. https://doi.org/10.1080/21505594.2016.1257457

15. Seyedmousavi, S.; Verweij, P. E.; Mouton, J. W. Expert Rev. Anti. Infect. Ther. 2015, 13, 9-27. https://doi.org/10.1586/14787210.2015.990382

16. Peyton, L. R.; Gallagher, S.; Hashemzadeh, M. Drugs Today (Barc) 2015, 51, 705-718. https://doi.org/10.1358/dot.2015.51.12.2421058

17. Chitasombat, M. N.; Kontoyiannis, D. P. Expert Opin. Pharmacother. 2015, 16, 1543-1558. https://doi.org/10.1517/14656566.2015.1057500

18. Revie, N. M.; Iyer, K. R.; Robbins, N.; Cowen, L. E. Curr. Opin. Microbiol. 2018, 45, 70-76. https://doi.org/10.1016/j.mib.2018.02.005

19. Perlin, D. S.; Rautemaa-Richardson, R.; Alastruey-Izquierdo, A. Lancet Infect. Dis. 2017, 17, 383392. https://doi.org/10.1016/S1473-3099(17)30316-X

20. Beardsley, J.; Halliday, C. L.; Chen, S.; Sorrell, T. C. Future Microbiol. 2018, 13, 1175-1191. https://doi.org/10.2217/fmb-2018-0059

21. Lopez-Ribot, J. L.; Wiederhold, N. P.; Patterson T. F., Fungal Drug Resistance: Azoles. In: Mayers, D.; Sobel, J.; Ouellette, M.; Kaye, K.; Marchaim, D. (eds), Antimicrobial Drug Resistance. Springer, Cham, 2017, Online ISBN 978-3-319-46718-4, https://doi.org/10.1007/978-3-319-46718-4_27

22. Caramalho, R.; Tyndall, J. D. A.; Monk, B. C. Sci Rep. 2017, 7, 15898. https://doi.org/10.1038/s41598-017-16123-9

23. Dannaoui, E. Int. J. Antimicrob. Agents 2017, 50, 617-621. https://doi.org/10.1016/j.ijantimicag.2017.08.010

24. Odds, F. C.; Brown, A. J. P.; Gow, N. A. R. Trends Microbiol. 2003, 11, 272-279. https://doi.org/10.1016/s0966-842x(03)00117-3 
25. Balding, P. R.; Porro, C. S., Munro, A. W.; Visser, S. P. J. Phys. Chem. A 2008, 112, 12911-12918. https://doi.org/10.1021/jp802087w

26. Zhang, Y.; Damu, G. L. V.; Cui, S. F.; Mi, J. L.; Tangadanchu, V. K. R.; Zhou, C. H. Med. Chem. Commun., 2017, 8, 1631-1639. https://doi.org/10.1039/C7MD00112F

27. Mast, N.; Zheng, W.; Stout, C. D.; Pikuleva, I. A. Molecular Pharmacology 2013, 84, 86-94; https://doi.org/10.1124/mol.113.085902

28. Kaushik, C. P.; Luxmi, R.; Kumar, M.; Singh, D.; Kumar, K.; Pahwa, A. Synth. Commun. 2019, 49, 118-128. https://doi.org/10.1080/00397911.2018.1544371

29. Thanh, N. D.; Hai, D. S.; Bich, V. T. N.; Hien, P. T. T.; Duyen, N. T. K.; Mai, N. T.; Dung, T. T.; Toan, V. N.; Van, H. T. K.; Dang, L. H.; Toan, D. N.; Van, T. T. T. Eur. J. Med. Chem. 2019, 167, 454-471. https://doi.org/10.1016/j.ejmech.2019.01.060

30. Fu, N.; Wang, S.; Zhang, Y.; Zhang, C.; Yang, D.; Weng, L.; Zhao, B.; Wang, L. Eur. J. Med. Chem. 2017, 136, 596-602, https://doi.org/10.1016/j.ejmech.2017.05.001

31. Aneja, B.; Irfan, M.; Kapil, C.; Jairajpuri, M. A.; Maguire, R.; Kavanagh, K.; Rizvi, M. M. A.; Manzoor, N.; Azam, A.; Abid, M. Org. Biomol. Chem. 2016, 14, 10599-10619. https://doi.org/10.1039/C6OB01718E

32. Dai, Z. H.; Chen, Y. F.; Zhang, M.; Li, S. K.; Yang, T. T.; Shen, L.; Wang, J. X.; Qian, S. S.; Zhu, H. L.; Ye, Y. H. Org. Biomol. Chem. 2015, 13, 477-486. https://doi.org/10.1039/C4OB01758G

33. Ramírez-Villalva, A.; González-Calderón, D.; Rojas-García, R. I.; González-Romero, C.; TamarízMascarúa, J.; Morales-Rodríguez, M.; Zavala-Segovia, N.; Fuentes-Benítes, A. Med. Chem. Commun. 2017, 8, 2258-2262, https://doi.org/10.1039/c7md00442g

34. González-Calderón, D.; Mejía-Dionicio, M. G.; Morales-Reza, M. A.; Ramírez-Villalva, A.; MoralesRodríguez, M.; Jauregui-Rodríguez, B.; Díaz-Torres, E.; González-Romero, C.; Fuentes-Benítes, A. Eur. J. Med. Chem. 2016, 112, 60-65. https://doi.org/10.1016/j.ejmech.2016.02.013

35. González-Calderón, D.; Mejía-Dionicio, M. G.; Morales-Reza, M. A.; Aguirre-de Paz, J. G.; RamírezVillalva, A.; Morales-Rodríguez, M.; Fuentes-Benítes, A.; González-Romero, C. Bioorg. Chem. 2016, 69, 1-6, https://doi.org/10.1016/j.bioorg.2016.09.003

36. Ballari, M. S.; Herrera-Cano, N.; Lopez, A. G.; Wunderlin, D. A.; Feresín, G. E.; Santiago, A. N. J. Agric. Food Chem. 2017, 65, 10325-10331. https://doi.org/10.1021/acs.jafc.7b04130

37. Brand, S.; Ko, E. J.; Viayna, E.; Thompson, S.; Spinks, D.; Thomas, M.; Sandberg, L.; Marco, M.; Miles, T. J.; Read, K. D.; Gilbert, I. H. J. Med. Chem. 2017, 60, 7284-7299. https://doi.org/10.1021/acs.jmedchem.7b00463

38. Shaikh, M. H.; Subhedar, D. D.; Nawale, L.; Sarkar, D.; Khan, F. A. K.; Sangshetti, J. N.; Shingate, B. B. Med. Chem. Commun. 2015, 6, 1104-1116. https://doi.org/10.1039/C5MD00057B

39. Kamal, A.; Rao, A. V. S.; Vishnuvardhan, M. V. P. S.; Reddy, T. S.; Swapna, K.; Bagul, C.; Reddy, N. V. S.; Srinivasulu, V. Org. Biomol. Chem. 2015, 13, 4879-4895. https://doi.org/10.1039/C5OB00232J

40. Irfan, M.; Alam, S.; Manzoor, N.; Abid, M. PLoS ONE 2017, 12, e0175710. https://doi.org/10.1371/journal.pone.0175710

41. Shaikh, M. H.; Subhedar, D. D.; Khan, F. A. K.; Sangshetti, J. N.; Shingate, B. B. Chin. Chem. Lett. 2016, 27, 295-301. https://doi.org/10.1016/j.cclet.2015.11.003

42. National Committee for Clinical Laboratory Standards Institute (CLSI), Document M38-A2: Reference Method for Broth Dilution Antifungal Susceptibility Testing of Filamentous Fungi, Approved Standard, Second Edition, Clinical and Laboratory Standards Institute, Wayne, PA, 2002.

43. Barry, A. L. An overview of the Clinical and Laboratory Standards Institute (CLSI) and its impact on antimicrobial susceptibility tests, in: Schwalbe, R.; Steele-Moore, L.; Goodwin, A.C. (Eds.), 
Antimicrobial Susceptibility Testing Protocols, CRC Press Taylor \& Francis Group, Florida, 2007, 1 6. ISBN: 9780824741006.

44. Espinel-Ingroff, A.; Canton, E. Antifungal susceptibility testing of filamentous fungi, in: Schwalbe, R.; Steele-Moore, L.; Goodwin, A.C. (Eds.), Antimicrobial Susceptibility Testing Protocols, CRC Press Taylor \& Francis Group, Florida, 2007, 209-241. ISBN: 9780824741006.

45. National Committee for Clinical and Laboratory Standards Institute (CLSI), M27-A3: Reference Method for Broth Dilution Antifungal Susceptibility Testing of Yeasts, Approved Standard, Third Edition, Clinical and Laboratory Standards Institute, Wayne, PA, 2008. ISBN: 1-56238-666-2.

46. Espinel-Ingroff, A.; Cantón, E. Antifungal susceptibility testing of yeasts, in: Schwalbe, R.; SteeleMoore, L.; Goodwin, A. C. (Eds.), Antimicrobial Susceptibility Testing Protocols, CRC Press Taylor \& Francis Group, Florida, 2007, 173-208. ISBN: 9780824741006.

47. Fothergill, A. W. Antifungal Susceptibility Testing: Clinical Laboratory and Standards Institute (CLSI) methods, in: G.S. Hall (Ed.), Interactions of Yeasts, Moulds, and Antifungal Agents. How to Detect Resistance, Springer Science-Business Media, 2012, 65-74. https://doi.org/10.1007/978-159745-134-5_2

48. Vigezzi, C.; Riera, F. O.; Caeiro, J. P.; Sotomayor, C. E. Rev. Argent. Microbiol. 2020, In Press, https://doi.org/10.1016/j.ram.2020.06.003

49. Choi, S.; Song, J. S.; Woo, J. H.; Kim, S. H. Mycoses 2019, 62, 1006-14. https://doi.org/10.1111/myc.12994

50. Aguirre-De Paz, J. G.; González-Calderón, D.; Fuentes-Benítes, A.; González-Romero, C. Tet Lett. 2018, 59, 1760-1762. https://doi.org/10.1016/j.tetlet.2018.03.075

51. Vargas-Herrera, N.; Saavedra-Velasco, M.; Pichardo-Rodriguez, R. Acta Méd. Peru. 2020, 36, 287290.

52. Valdés, T. G. E.; Martínez, B. M. E.; Morayta, R. C. A. R. R. Rev. Latin. Infect. Pediatr. 2020, 33, 4956. https://doi.org/10.35366/92386 